\title{
Compensatory strategies following visual search training in patients with homonymous hemianopia: an eye movement study
}

\author{
Sabira K. Mannan • Alidz L. M. Pambakian • \\ Christopher Kennard
}

Received: 10 November 2009/Accepted: 24 May 2010/Published online: 16 June 2010

(C) The Author(s) 2010. This article is published with open access at Springerlink.com

\begin{abstract}
A total of 29 patients with homonymous visual field defects without neglect practised visual search in 20 daily sessions, over a period of 4 weeks. Patients searched for a single randomly positioned target amongst distractors displayed for $3 \mathrm{~s}$. After training patients demonstrated significantly shorter reaction times for search stimuli (Pambakian et al. in $\mathbf{J}$ Neurol Neurosurg Psychiatry $75: 1443-1448,2004)$. In this study, patients achieved improved search efficiency after training by altering their oculomotor behaviour in the following ways: (1) patients directed a higher proportion of fixations into the hemispace containing the target, (2) patients were quicker to saccade into the hemifield containing the target if the initial saccade had been made into the opposite hemifield, (3) patients made fewer transitions from one hemifield to another before locating the target, (4) patients made a larger initial saccade, although the direction of the initial saccade did not change as a result of training, (5) patients acquired a larger visual lobe in their blind hemifield after training. Patients also required fewer saccades to locate the target after training reflecting improved search efficiency. All these changes were confined to the training period and maintained at follow-up. Taken together these results suggest that visual training facilitates the development of specific compensatory eye movement strategies in patients with homonymous visual field defects.
\end{abstract}

S. K. Mannan $(\bowtie) \cdot$ C. Kennard

Department of Clinical Neurology, John Radcliffe Hospital,

University of Oxford, Oxford OX3 9DU, UK

e-mail: sabira.mannan@clneuro.ox.ac.uk

S. K. Mannan - A. L. M. Pambakian - C. Kennard Centre for Neuroscience, Imperial College London, Charing Cross Campus, London W6 8RP, UK
Keywords Homonymous hemianopia · Visual search · Training $\cdot$ Eye movements $\cdot$ Rehabilitation

\section{Introduction}

Homonymous hemianopia $(\mathrm{HH})$, the loss of vision in one half of the visual field, results from unilateral lesions of the geniculostriate pathway. Although a degree of spontaneous recovery may occur in some patients with $\mathrm{HH}[1-4]$ it is rarely sufficient to remove the disabling consequences of visual field loss-difficulties in reading, driving and visual exploration, and deficits of visuo-spatial orientation [5, 6]. Although a small minority of patients with hemianopia appear to retain sensitivity to briefly flashed or moving stimuli within their blind field [7], this residual vision appears inaccessible to conscious perception and action.

Rehabilitation of patients with $\mathrm{HH}$, once thought an untreatable condition, has recently become a matter of intense debate (see [8] for a review). Several groups have claimed restitution of visual function and consequent visual field enlargement by repeated visual stimulation, usually at the border of the scotoma [9-15] and changes in cortical representation in the pre-striate cortex as a result of training have also been reported [16]. Other studies have found no effect of training on visual field enlargement [17-20]. However, rigorous control of fixation during training eliminates significant visual field enlargement [20], an observation which has led to controversy over the very existence of visual field enlargement [14, 21-23].

An alternative rehabilitation technique is visual search training, which aims to adapt patients' scanning strategies to most effectively compensate for their visual field loss. In some cases, HH disrupts normal scanning patterns with patients exhibiting disorganised scanpaths with high rates 
of refixation and inaccurate saccades [24] and it has been proposed that the loss of re-entrant pathways from higher visual areas to the damaged striate cortex may result in uncertainty about spatial locations across saccades [3]. Some patients make a series of small saccades, with long latencies into their blind field [25-27]. In light of these scanpath abnormalities, patients are often trained to make systematic horizontal or vertical scanning saccades into their blind field (oculomotor training) [5, 24, 27-29]. Training usually leads to an enlargement of the region in which subjects can successfully locate a target with eye movements (the visual search field, VSF) and a reduction in response times [5, 29-31]. Importantly, training also leads to improvements in activities of daily living (ADLs), assessed both empirically as well as with subjective questionnaires [24, 28-30]. However several questions concerning visual search training remain unresolved. Firstly, most patients, even without training, learn to compensate for their field loss by making more fixations and spending a greater proportion of viewing time in their "blind" halffield [24, 32-34] and there is evidence that this compensatory strategy evolves over time [26]. Does specific training therefore simply reinforce these naturally occurring strategies or does it, in fact, further alter the efficacy of search eye movements? Secondly, as repeated visual search practise leads to perceptual learning can the reductions in response times associated with visual search training be explained by neuronal changes in response to the learnt stimulus features, rather than changes in eye movement strategies? Several recent studies have attempted to answer these questions by evaluating oculomotor behaviour in patients with $\mathrm{HH}$ following oculomotor training $[35,36]$ or training on extended visual search containing multiple targets [37]. By contrast, Pambakian et al. [30] trained 29 patients with homonymous visual field defects to perform visual search without prescribing any particular oculomotor strategy to patients, reasoning that repeated visual search practise with search durations of $3 \mathrm{~s}$ or less would allow patients to develop their own adaptive scanpaths [38]. As a group, patients in this study evinced larger VSF after training, together with a significant mean reduction of response times. The focus of the current paper is to characterise the changes in oculomotor scanning that resulted from training.

\section{Subjects and methods}

\section{Subjects}

A total of 31 patients (16 female) with visual (18 left; 13 right) field defects, ranging from homonymous quadrantanopia to complete hemianopia, due to post chiasmatic lesions, were recruited (mean age $=46.9$ years, range 24 75 years). High resolution structural MRI scans revealed that 22 had cerebral infarctions, 6 had arterio-venous malformations (AVM) which had bled, one had a temporal lobectomy, one had a grade 2 glioma in the occipatal lobe and one had a cystic teratoma. Patients were recruited at least 3 months after the onset of their hemianopia and were aware of their field defects. Visual acuities were corrected to $6 / 6$ using trial or contact lenses. None of the patients had co-existing eye movement pathology, abnormalities on fundoscopy or slit lamp examination, visual neglect [40] or reduced mini mental test scores. All patients gave informed written consent. This study was approved by the local Riverside ethics committee.

\section{Experimental procedure}

\section{Training procedure}

Patients were trained on a dedicated 21 inch TV monitor $\left(25^{\circ} \times 10^{\circ}\right)$, installed in a quiet darkened room in the patient's own home and used under supervision. Patients searched for a single randomly positioned target element (either lines, squares or triangles) that differed from 40 homogenous distractors by a single feature (size or orientation). Prior to each search, patients initially fixated a central cross for $1 \mathrm{~s}$ to ensure that their gaze commenced at the centre of the screen. Search stimuli were presented for a maximum of $3 \mathrm{~s}$ and subjects indicated target absence/ presence using a two-button mouse response pad. Subjects were instructed to detect and fixate the target as accurately and rapidly as possible, but to keep head movements to a minimum. Non-target trials $(20 \%)$ were randomly interspersed to determine response accuracy. Patients performed 600 trials in one 40-min session, in blocks of 100 trials. Patients received 5 daily sessions per week totalling 20 sessions during the month of training.

\section{Testing procedure}

Patients were tested on five separate occasions (referred to as visits $0-5$ ), each a month apart. Visit 0 served as a practise visit and the data for this visit were discarded. In this study each patient was tested twice before (visit 1 and 2) and twice after (visit 3 and 4) they were trained. Changes between visits 1 and 2 reflected spontaneous recovery or practice effects as no training occurred during this period. Changes between visits 2 and 3 represented the true effects of training. The fourth visit was included to determine whether the treatment effects were robust and would be maintained after training.

On each visit patients performed 200 visual search trials for a single randomly positioned target line, oriented either 
at $15^{\circ}, 30^{\circ}$ or $45^{\circ}$ amongst vertical distractors $(n=40)$. These stimuli were chosen to reflect a continuum of search difficulty from inefficient to efficient search. The target had equal probability of appearing in either visual hemifield. Null trials constituted $25 \%$. Stimuli were generated on a high resolution 20 inch monitor, subtending $20^{\circ} \times 20^{\circ}$. Eye movements were recorded using the P-scan system, a binocular, video-based infrared device with $50 \mathrm{~Hz}$ sampling rate and 0.2 spatial resolution [39]. Subjects' heads were clamped at the temples and placed on a chin rest to minimise head movements. Stimulus presentation and subject instructions were identical to those for the training paradigm.

\section{Analysis of eye movements}

Only target present trials were considered. Fixation was defined as a point where the eye position varied less than $0.5^{\circ}$ over a minimum of $60 \mathrm{~ms}$. Two successive fixations required mean positions at least $0.5^{\circ}$ apart [41]. Trials in which subjects made false negative responses or failed to respond within $3 \mathrm{~s}$ were excluded, as were trials in which the initial fixation did not fall within $1^{\circ}$ of the central fixation cross or no fixations fell within $2^{\circ}$ of the target. Three-way repeated measures analysis of variances (ANOVA, SPSS version 9), using visit (four visits, two pre- and two post-training), target position (target located in the blind vs. seeing field) and target type $\left(15^{\circ}, 30^{\circ}, 45^{\circ}\right)$ as the factors, were employed to analyse the following oculomotor parameters: fixation duration, saccade amplitude and numbers of saccades. Directional and hemispace analysis was also considered. Directional analysis was performed on the number of saccades made in the direction of the hemianopic and intact hemifields. In order to perform hemispace analysis the midline of the screen was taken as the dividing line between hemianopic and intact hemispace.

Visual lobe is commonly defined as the area within which a target can be fixated within a single saccade. Lobe size is a function of the characteristics of the target and background and, in the case of patients with $\mathrm{HH}$, will be highly asymmetric, with a constricted visual area in the blind hemifield. Here visual lobe was defined as the area within which the target was fixated during the next saccade and was therefore defined by the amplitude and direction of the saccade that led to fixation on target. For each patient, the direction and amplitude of the saccade that landed on the target in each trial was logged. Every such saccade was placed into a direction bin, $\Theta$, such that the first bin included saccades of direction $-15^{\circ} \leq \Theta<15^{\circ}$, the second bin included saccades with direction $15^{\circ} \leq \Theta<45^{\circ}$ and so on. The median amplitude of saccades within a bin was obtained, and a vector of this amplitude and direction was drawn from the origin. The area within the polygon produced by connecting the endpoints of successive vectors delineated the area of the visual lobe. To calculate the two parts of the visual lobe-the visual lobe area within the blind field and the area within the seeing field - a vertical line was drawn through the lobe passing through the origin.

In all analyses a significance level was set at $p<0.05$.

\section{Results}

Drop-outs

Of the 31 patients recruited into the study, 29 completed the training. One patient was unable to keep her head sufficiently still for the requirements of eye movement recording due to spasmodic torticollis and the other patient dropped out due to social circumstances.

\section{Oculomotor strategy: strategic placement of fixations}

There was no main effect of visit on the proportion of initial saccades made into the hemianopic field $[F(3,28)>2$, n.s.], patients making exactly the same proportion $(50 \%)$ before and after training (Table 1a). There was a main effect of target position on the direction of the first saccade with a higher proportion of initial saccades directed towards, rather than away from, the hemifield containing the target $[F(1,28)=16.7, p<0.001$, Cohen's $d=1.50]$.

The proportion of fixations made in hemianopic hemispace also remained unchanged after training $[F(3,28)<1.0$, n.s.], i.e. patients making the same proportion (47\%) before and after training. There was a significant effect of target position and a significant interaction between the position of the target and visit $[F(3,224)=2.9, p<0.05]$. Planned comparisons reveal a subtle effect of training on fixation positions; patients made a significantly greater proportion of fixations in hemianopic hemispace after training when the target was located on this side (Fig. 1a). They also made a significantly greater proportion of fixations in intact hemispace after training when the target was also located in intact hemispace $[F(3,224)=4.62, p<0.05$, Cohen's $d=0.95]$. By contrast, there was no significant difference between visits 3 and $4[F(3,224)=0.36$, n.s.], suggesting that the change was maintained after training, and between visits 1 and 2 the proportion of fixations falling in the hemispace containing the target actually decreased by $3 \%$-opposite to the effect of training $[F(3,224)=4.5$, $p<0.05$, Cohen's $d=-0.38$ ].

Training clearly facilitates the direction of saccades into the hemifield containing the target. But how do patients learn to selectively saccade to the appropriate hemispace? 
Table 1 Strategic placement of fixations and fixation durations. The mean values of oculomotor variables relating to strategy are shown

\begin{tabular}{|c|c|c|c|c|c|}
\hline & $\begin{array}{l}\text { Position of target } \\
\text { (hemianopic/intact } \\
\text { hemispace) }\end{array}$ & Visit 1 & Visit 2 & Visit 3 & Visit 4 \\
\hline \multirow[t]{2}{*}{ (A) Proportion of initial saccades made into the hemianopic field (\%) } & Hemianopic & $54(3)$ & $51(2)$ & $51(3)$ & $56(3)$ \\
\hline & Intact & $49(3)$ & $48(2)$ & $48(3)$ & $52(3)$ \\
\hline \multirow[t]{2}{*}{ (B) Latency of the initial saccade (ms) } & Hemianopic & $305(17)$ & $282(10)$ & $263(9)$ & $263(8)$ \\
\hline & Intact & $285(13)$ & $266(9)$ & $250(8)$ & $248(8)$ \\
\hline \multirow[t]{2}{*}{ (C) Mean duration (ms) for fixations made in hemainopic hemispace only } & Hemianopic & $194(13)$ & $197(11)$ & $189(10)$ & $188(7)$ \\
\hline & Intact & $149(13)$ & $171(11)$ & $152(9)$ & $144(10)$ \\
\hline \multirow[t]{2}{*}{ (D) Mean duration (ms) for fixations made in intact hemispace only } & Hemianopic & $190(7)$ & $191(4)$ & $188(7)$ & $189(7)$ \\
\hline & Intact & $179(9)$ & $180(8)$ & $163(8)$ & $183(10)$ \\
\hline \multirow[t]{2}{*}{ (E) Mean duration (ms) for fixations made before switch } & Hemianopic & $210(12)$ & $204(6)$ & $198(12)$ & $198(12)$ \\
\hline & Intact & $228(9)$ & $232(11)$ & $178(7)$ & $189(7)$ \\
\hline
\end{tabular}

Standard error of the mean, SEM in parentheses
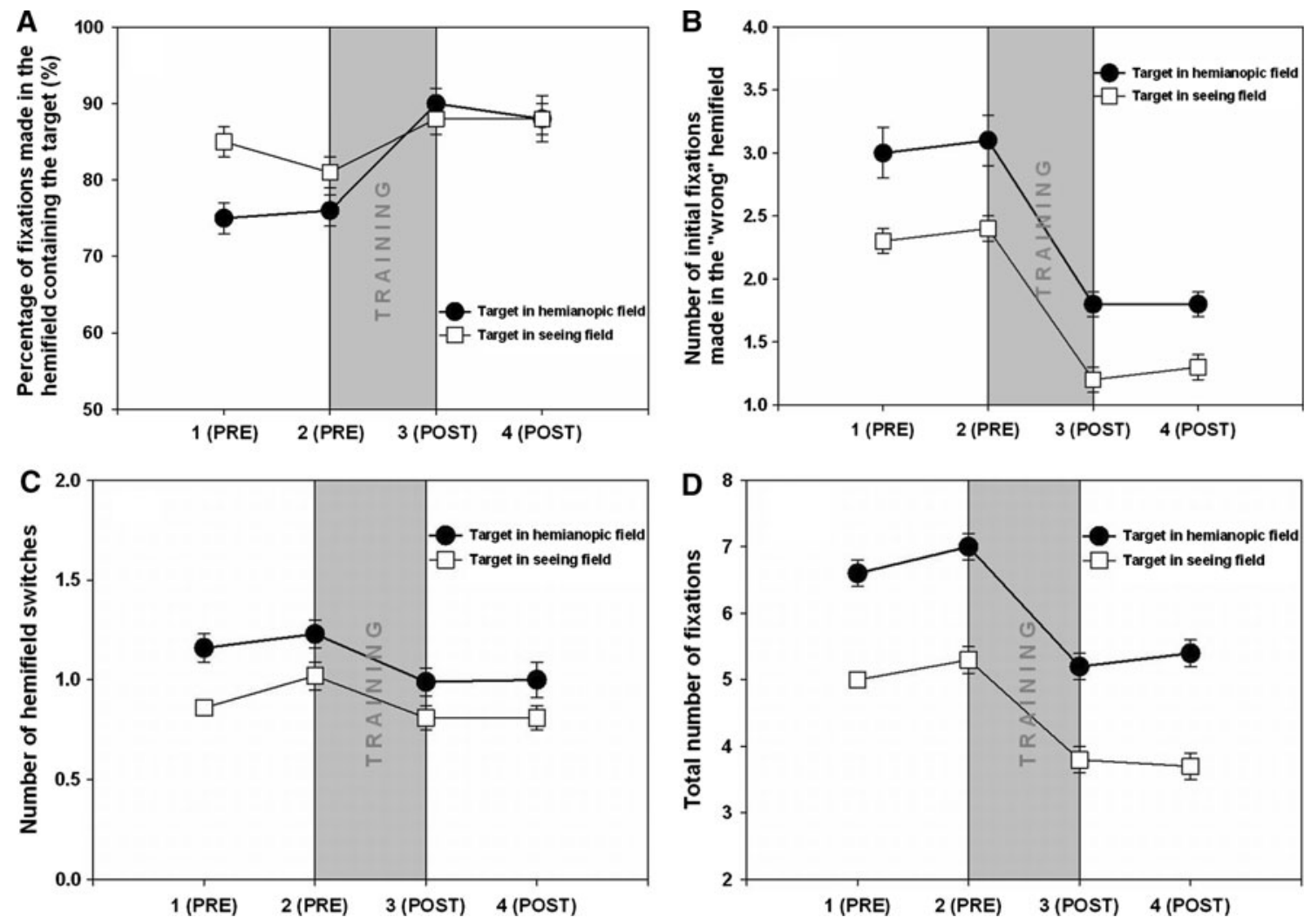

Fig. 1 Effect of training on fixation search parameters in four sessions each 1 month apart ( 1 baseline, 2 second baseline immediately preceding training, 3 immediately following 1 month training, 41 month after cessation of training. a The percentage of fixations made in the hemifield containing the target increased after training. b The number of fixations initially made in the hemifield not

containing the target decreased after training. c The number of hemifield switches made before locating the target decreased after training. d Total number of fixations made during search decreased after training. Filled circles denote target in the blind hemifield and white squares denote target in the seeing hemifield. Error bars show 1 SEM

In order to investigate this we considered only those trials in which subjects made an initial saccade into the "wrong" hemifield, i.e. the hemifield not containing the target, and established how many fixations were made in the wrong

hemifield before "switching" to the correct one. There was a main effect of visit on the number of fixations made before switching $[F(3,28)=3.4, \quad p<0.05$; Fig. 1b]. Planned comparisons revealed that patients made fewer 
initial fixations in the wrong hemispace before switching as a result of training $[F(3,28)=7.83, p<0.01$, Cohen's $d=0.90]$. This decrease was specific to the training period; there was no change between visits 1 and 2 $[F(3,28)=2.71$, n.s. $]$ and it was maintained after training $[F(3,224)=1.12$, n.s.]. There was also a main effect of target position, with patients making more fixations in the "wrong" hemispace when the target actually lay in intact rather than hemianopic hemispace $[F(1,28)<15.3$, $p<0.001$, Cohen's $d=0.78]$.

We then considered the number of times patients switched hemifields before locating the target. There was a main effect of visit on the number of hemifield switches $[F(3,28)=12.5, p<0.001 ;$ Fig. $1 \mathrm{c}]$. Planned comparisons revealed that patients made significantly more hemifield switches at visit 2 compared to visit $1[F(3,28)=10.4$, $p<0.005$, Cohen's $d=-0.13]$. In contrast, patients required fewer hemifield switches after training $[F(3,28)=$ 56.23, $p<0.001$, Cohen's $d=1.85$ ]. There was no further change between visits 3 and 4 , suggesting that the decrease was maintained after training $[F(3,224)=1.49$, n.s.]. Thus, although training has no effect on the likelihood of patients making an initial saccade to the correct hemifield, patients were less likely to "switch out" from the correct hemifield, once it had been reached, after training. There was also a main effect of target position with patients making more switches when the target was in hemianopic rather than in intact hemispace $[F(1,28)<9.9, p<0.004$, Cohen's $d=0.59$ ].

\section{Summary}

After training patients made a higher proportion of fixations in the hemispace containing the target. In trials in which patients made their initial saccade into the hemifield not containing the target, they became faster at switching to the hemifield containing the target after training, i.e. making fewer fixations in the "wrong" hemifield before switching. After training patients also made fewer transitions from one hemispace to another before locating the target. Training, however, had no effect on the direction of the initial saccade.

\section{Numbers of fixations and fixation durations}

There was a significant main effect of visit on the number of fixations made during search $[F(3,28)=10.65, p<0.001$; Fig. 1d]. Planned comparisons revealed that patients made significantly more fixations at visit 2 compared to visit 1 $[F(3,28)=17.8, p<0.001$, Cohen's $d=-0.48]$ but that after training patients made significantly fewer fixations compared to both visits 1 and $2[F(1,28)=5.1, p<0.05$, Cohen's $d=1.28 ; F(3,28)=19.67, p<0.001$, Cohen's $d=1.56]$. This decrease in fixation numbers was maintained 1 month after training $[F(3,28)=0.1$, n.s.]. There was also a main effect of target position with patients making more fixations when the target was in hemianopic rather than intact hemispace $[F(1,28)=127.4, p<0.001$, Cohen's $d=1.51]$.

Although there was a significant main effect of visit on the latency of the first saccade $[F(3,28)=7.9, p<0.001]$, this was not specific to the training period (Table $1 b$ ). Planned comparisons revealed that patients were faster at initiating a saccade during the second visit compared to the first, reflecting the effect of spontaneous improvement or practise in the absence of any training $[F(3,28)=6.7$, $p<0.05$, Cohen's $d=0.32$ ]. There was a further decrease in initial latency between visit 2 and visit $3[F(3,28)=4.3$, $p<0.05$, Cohen's $d=0.38$ ], the training period, and no further decrease between visits 3 and $4[F(3,28)=0.1$, n.s.]. Initial saccadic latencies were shorter when the target was in intact rather than hemianopic hemispace $[F(1,28)=21.1, p<0.001$, Cohen's $d=0.30]$.

Mean durations of fixations made in the hemianopic hemispace did not change duration as a result of training $[F(3,28)=2.27$, n.s.; Table 1c]. In contrast, for fixations made in intact hemispace (Table 1d), while there was no significant main effect of visit $[F(3,28)=1.41$, n.s.], there was a significant main effect of position of the target $[F(1,28)=11.39, p<0.001$, Cohen's $d=0.34]$, and a significant interaction between visit and the position of the target $[F(3,224)=3.0, p<0.05]$. Planned comparisons revealed that training resulted in quicker fixation durations in intact hemispace if the target was also on the intact side of the screen $[F(3,224)=6.5, p<0.02$, Cohen's $d=0.40]$, but not if the target was on the hemianopic side of the screen $[F(3,224)<2$, n.s.]. Interestingly, this specific decrease in the duration of fixations made in intact hemispace for trials containing targets in intact hemispace disappeared 1 month after training $[F(3,224)=5.0$, n.s. $]$.

Finally we considered only those trials in which patients initially saccaded to the incorrect hemifield (the hemifield not containing the target). There was a significant interaction between target position and visit $[F(3,224)=2.9$, $p<0.05$; Table 1e] for mean fixation durations made before the switch to the correct hemifield. Planned comparisons revealed that patients made quicker fixations in the incorrect hemianopic hemispace before saccading to the target-containing intact space after training $[F(3,224)=2.9, p<0.05$, Cohen's $d=1.11]$. There was no change between visits 1 and $2[F(3,224)=2.1$, n.s. $]$ and the decrease was maintained after training $[F(3,224)=1.2$, n.s.]. By contrast, there was no change in durations for fixations initially made in the incorrect intact hemispace $[F(3,224)<2$, n.s. $]$. 
Table 2 Saccade amplitude and saccade direction

\begin{tabular}{llllll}
\hline & $\begin{array}{l}\text { Position of target } \\
\text { (hemianopic/intact } \\
\text { hemispace) }\end{array}$ & Visit 1 & Visit 2 & Visit 3 & Visit 4 \\
\hline (A) Mean amplitude of all saccades $\left(^{\circ}\right)$ & Hemianopic & $6.5(0.3)$ & $6.6(0.3)$ & $6.8(0.3)$ & $6.8(0.3)$ \\
& Intact & $6.2(0.2)$ & $5.7(0.3)$ & $4.8(0.2)$ & $4.6(0.3)$ \\
$\begin{array}{llll}\text { (B) Proportion of saccades made } \\
\text { in a contra-lesional direction }(\%)\end{array}$ & Hemianopic & $56(2)$ & $56(2)$ & $57(2)$ & $25(2)$ \\
\hline
\end{tabular}

The mean values are shown ( $\mathrm{SE}$ in parentheses)

\section{Summary}

After training patients required fewer fixations to reach the target. Fixations made in intact hemispace in trials in which the target was also in intact hemispace became significantly shorter after training. This effect disappeared 1 month after training. Patients became quicker at fixating locations in hemianopic hemispace-when the target was actually in the opposite hemifield—after training.

Saccade amplitude and saccade direction

There was a significant main effect of visit on the mean amplitude of the initial saccade made by the patients $[F(3,28)=3.16, p<0.001 ;$ Fig. 2a]. Planned comparisons revealed that patients made a significantly larger initial saccade after training [visit $2=6.27^{\circ}$ cf visit $3=6.81^{\circ}$; $F(3,28)=9.78, p<0.004$, Cohen's $d=0.30]$. In contrast there was no significant difference between visits 1 and 2 $[F(3,28)=0.1$, n.s. $]$ and visits 3 and $4[F(3,28)=2.1$, n.s.]. There was a main effect of target position with patients making longer initial saccades when the target was in the intact rather than the hemianopic field $[F(1,28)=$ 5.57, $p<0.05$, Cohen's $d=0.22$ ].

The mean amplitude of saccades following the initial saccade showed no effect of training $[F(3,28)=2.24$, n.s.; Table 2a] although there was a main effect of target position with patients making longer saccades when the target lay in hemianopic rather than intact hemispace $[F(1,28)=$ $34.2, p<0.001$, Cohen's $d=1.00]$. We next considered the proportion of saccades made in the hemianopic vs intact direction. There was a main effect of target position on this ratio, with patients making $57 \%$ of their saccades in a hemianopic direction if the target was in hemianopic hemispace $[F(1,28)=210.0, p<0.001$, Cohen's $d=2.95$; Table 2b], while only $25 \%$ saccades were in a hemianopic direction for targets in intact hemispace. There was no main effect of visit $[F(3,28)=0.39$, n.s.] but there was a significant interaction of visit and target position $[F(1,28)=3.0, p<0.05]$. Planned comparisons revealed that whilst the proportion of contralesional saccades for targets in the intact field fell slightly after training, the proportion of such saccades rose slightly for targets in the hemianopic field $[F(3,28)=2.71, p<0.05$, Cohen's $d=0.19]$.

The visual lobe area in the seeing field showed no effect of training $[F(3,28)=2.24$, n.s.; Fig. 2b]. By contrast, the area of the visual lobe in the blind field increased significantly after training $[F(3,28)=5.08, p<0.05$, Cohen's $d=0.81$; Fig. 2c]. There was no change between visits 1 and $2[F(3,28)<2$, n.s. $]$ and the increase was maintained after training $[F(3,224)<2$, n.s. $]$. This slightly surprising result suggests that, as a result of training, patients are able to accurately find and fixate targets within a larger area of their blind field within a single saccade.

\section{Summary}

After training patients made a longer initial saccade although the mean amplitude of subsequent saccades did not change with training. After training patients made a higher proportion of saccades in the direction of the target, i.e. patients made more contralesional than ipsilesional saccades when the target was in hemianopic hemispace and, conversely, more ipsilesional than contralesional saccades when the target was intact hemispace. After training, patients were able to locate targets within a larger area of their blindfield.

\section{Discussion}

Visual search training in a group of 29 patients with homonymous hemianopia led to a number of trainingspecific changes in saccadic behaviour accompanied by significant reduction in the mean search times required to locate a single target amongst distractors [30]. After visual search training patients made a higher proportion of fixations into the hemispace containing the target; patients were quicker to switch into the hemifield containing the target if the initial saccade had been made into the opposite hemifield; patients made fewer transitions from one hemifield to another before locating the target; patients made a larger initial saccade, although the direction of the 

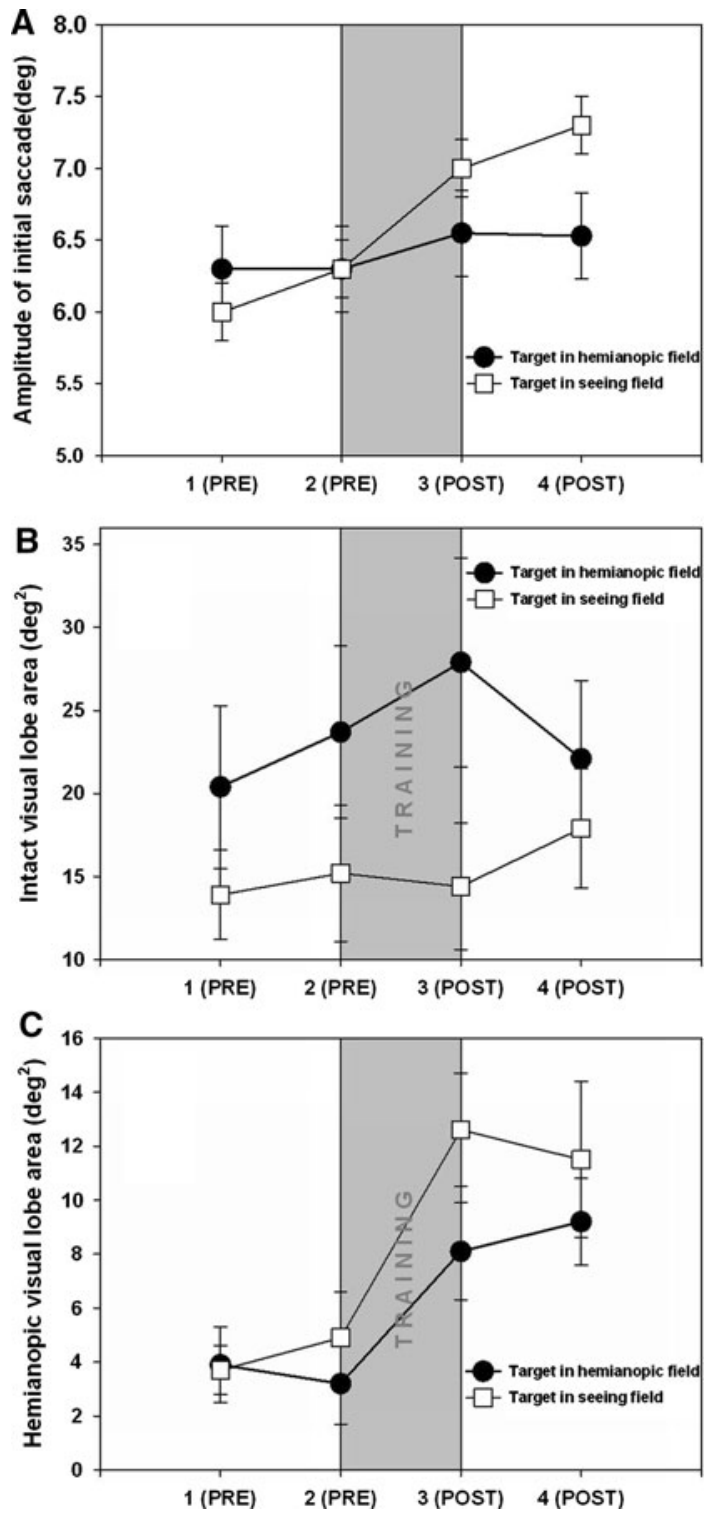

Fig. 2 Effect of training on fixation search parameters in four sessions each 1 month apart ( 1 baseline, 2 second baseline immediately preceding training, 3 immediately following 1 month training, 4 1 month after cessation of training. a The mean amplitude of the initial saccade increased after training. b The mean area of the intact visual lobe - the area within the seeing field in which the patient can fixate the target in the following saccade-remained unchanged after training. c The mean area of the hemianopic visual lobe- the area within the hemianopic field in which the patient can fixate the target in the following saccade-increased after training. Filled circles denote target in the blind hemifield and white squares denote target in the seeing hemifield. Error bars show 1 SEM

initial saccade did not change, and there was a very small but significant increase in the number of saccades made in the direction of the target after training. In addition, patients required fewer saccades to locate the target after training, reflecting increased search efficiency and the area within which targets could be fixated in the blind field also increased. These changes were specific to the period of training, i.e. there was no spontaneous change or improvement between visits 1 and 2 (the visits prior to the training phase). Moreover all the changes seen after training at visit 3 were maintained at visit 4 (a month after the training had been completed).

Previous visual search rehabilitation programs designed to treat deficits in visual exploration have primarily aimed at improving disorders of visuo-spatial disorientation apparent in some patients with HH. Thus these training programs require patients to search for a number of targets over an extended time period and have explicitly instructed patients to make large initial saccades into the hemianopic hemifield, and to search in a rigid systematic manner [5, $24,28,31,32,35,36]$ or through extended visual search training [37]. By contrast we trained [30] subjects to search for a single target for $3 \mathrm{~s}$ or less, and did not suggest any particular oculomotor behaviour. Schuett et al. [34] have shown that healthy volunteers with simulated hemianopias adopt spontaneous oculomotor compensatory strategies following a very short single period of visual search training [34]. Although healthy volunteers with simulated hemianopias cannot be directly compared to patients with brain damage, our results demonstrate that patients, left to themselves, also adopt a subtle and effective visual search strategy, in our case, after a prolonged period of training.

The efficacy of visual search training in patients with HH depends critically on whether improvements in visual search generalise from the stimuli used in training to everyday visual search tasks. While one recent study found that visual search training resulted in compensatory oculomotor scanning in a picture viewing task [37], a study of simulated $\mathrm{HH}$ in healthy volunteers found no evidence of transfer between tasks [38]. Another recent study of oculomotor training in patients with $\mathrm{HH}$ resulted in adaptive changes during visual search, only if the oculomotor training involved audio-visual, rather than visual stimuli [35] in contrast to our study, where visual search training without auditory cues was sufficient to produce compensatory eye movements.

A related question is whether the changes in oculomotor scanning reflect genuine changes in oculomotor strategy or are consequent upon neuronal tuning or neuronal plasticity in the visual cortex. For example, visual functions such as stereoacuity, orientation and motion detection, segmentation of textures and hyperacuity improve after prolonged practise $[42,43]$. Because learning for these visual features tends to be very specific, often confined to the trained visual field location, or trained orientation or motion, with little transfer to the untrained eye, low-level changes in neuronal tuning, e.g. in V1, have been implicated. In contrast, perceptual learning in visual search has been shown to transfer over retinal locations, and from the 
trained to the untrained eye. Some studies have shown no transfer of learning to novel visual search tasks [42, 43], whereas others studies have found far less specificity [4447]. These latter authors suggest that training in visual search initially involves neuronal changes in regions high in the visual brain-for example, practise in orientation discrimination leads to narrower orientation tuning curves in macaque V4-and involve changes in the deployment of visuo-spatial attention. Some of the oculomotor behaviour documented here may result from perceptual learning. For example fixation durations in the intact hemispace are shorter after training. One interpretation of this finding is that visual search practise results in perceptual learning, facilitating target detection prior to-and during-fixation on target (hence lower fixation durations). Fixation durations for targets in hemianopic space remain unchanged as low-level perceptual learning cannot occur in the damaged striate cortex. The effect of this postulated perceptual learning disappears once visual search training has ceased.

Other findings within our study suggest the existence of cognitive changes in strategy. We found that the mean saccadic amplitude did not increase after training, as would be expected if neuronal changes resulted in improved tuning specific to the search stimuli. Patients also learnt to saccade more quickly into the hemianopic hemifield when the target was also in the hemianopic field-even though their $\mathrm{HH}$ would prevent them detecting these targets. The visual lobe size - the area within the blind field within which patients could saccade successfully to the targetalso increased after training. This may appear surprising as visual field perimetry demonstrated no increase in visual fields after training [30]. However the increasing lobe size may simply reflect more efficient placement of the eyes after training, allowing patients to saccade to the probable location of the target. The notion that visual search improvements are strategically based is also supported by the finding [30] that patients demonstrated significant improvements, on the order of a $25 \%$ reduction in response times, for tasks of daily living involving novel visual search stimuli.

The scanning behaviour of patients during search demonstrates a close interplay between bottom-up visual influences and top-down strategic ones. So, for example, the proportion of fixations made in hemianopic hemispace and the proportion of initial saccades made into the hemianopic hemifield were significantly higher than $50 \%$ only when the target was in hemianopic hemispace. This contrasts with hemianopic scanning for natural scenes [26], where we found that patients with long-standing hemianopias, indeed made more fixations and spent a greater proportion of their viewing time in hemianopic hemispace. In that study hemifield differences were accentuated when images were filtered to remove much of the semantic and visual content of the scene, and the authors suggested that top-down cognitive adaptive strategies play an increasing role in directing eye movements in the absence of semantic/visual information. In a carefully controlled, randomized study Roth et al. [37] found that patients altered their eye movement patterns following visual search therapy, placing a higher proportion of their fixations in the blind half field during free scene inspection, even if the salient object within the scene was located on the seeing side. It thus appears that strategic guidance of eye movements is task specific; in the current study, where subjects were required to search for a target equally likely to appear in either hemifield, patients did not automatically bias their eye movements to hemianopic hemispace-thereby automatically rendering eccentric ipsilesional targets more difficult to detect-and, instead, adopted a more advantageous strategy of more rapid switching of hemifields as a result of training. Homonymous visual field defects are a common consequence of stroke; $30 \%$ of all patients with stroke [48] and $70 \%$ of patients with stroke involving the posterior cerebral artery have such field defects [49]. Homonymous visual field defects are associated with a poor prognosis for recovery [50-53], particularly when combined with visual hemispatial neglect [54-57] and yet there are still no established rehabilitation programs for such patients. The efficacy visual restoration therapy is still subject to consideration [20-23] and requires 6 months of training. Visual restoration studies either involve repeated stimulation at the borders of a scotoma or deep within the blind field with the aim of stimulating extra-striate cortex. Both methods can generate unwanted eye movements [11, 18, 20-22]. In fact, where stimuli are repeatedly presented in the blind field for $>0.5 \mathrm{~s}$ durations during training, part of the "restitution" may actually result from patients learning efficient oculomotor strategies, as patients did here, and that allow them to localise the target within the presentation time [16, 58, 59].

Here we have shown that visual search training for short stimulus durations, results in effective adaptive oculomotor strategies. The results of this paper complement an earlier paper [30] in which we demonstrated the transfer of visual search training to activities of daily living and significant subjective improvements in the same patients. The visual search training method described here is short (1 month duration), inexpensive, and can be conducted by patients in their own homes without the intervention of a therapist. Importantly, however, our present study cannot disentangle the effects of training from the potential role of spontaneous recovery. A future study with a hemianopic control group undergoing testing in four sessions but no training would be required to rule out the role of spontaneous recovery. Spontaneous oculomotor adaptation in patients with homonymous hemianopia is very well documented 
$[25,27,33,57]$ although hemianopic patients with additional damage to occipito-parietal cortex or posterior thalamus appear less likely to spontaneously adopt compensatory oculomotor strategies [24]. An earlier small cross-sectional study of hemanopic patients suggests a relatively long time-course for spontaneous recovery which takes 6 months to develop and may continue to evolve for over a year [26]. Although the majority $(72 \%)$ of our patients were recruited $>1$ year after onset, it is possible that spontaneous adaptation could coincide with the critical month of training between sessions 2 and 3, but not occur during sessions 1 and 2. In response to clinical need, however, a DVD of the entire visual search program is available at http://vision.metope.org. And in support of our findings, a recent study where patients were randomly assigned to a visual search training or visual restitution training group found that only patients in the former group demonstrated oculomotor changes and subjective benefits in activities of daily living [37].

Acknowledgments We thank the patients who participated and the staff of the stroke units at Charing Cross Hospital. This research was supported by grants from the Wellcome Trust to CK and the Stroke Association to ALMP (5/97). We thank Dr Stephen Hicks for programming the visual search stimuli into DVD format.

Open Access This article is distributed under the terms of the Creative Commons Attribution Noncommercial License which permits any noncommercial use, distribution, and reproduction in any medium, provided the original author(s) and source are credited.

\section{References}

1. Hier DB, Mondlokc J, Caplan L (1983) Recovery of behavioural abnormalities after right hemisphere stroke. Neurology 33:675679

2. Zihl J, von Cramon D (1979) Restitution of visual function in patients with cerebral blindness. J Neurol Neurosurg Psychiatry 42:312-322

3. Zihl J, von Cramon D (1985) Visual field recovery from scotoma in patients with postgeniculate damage: a review of 55 cases. Brain 108:439-469

4. Tiel K, Kolmel HW (1991) Patterns of recovery from homonymous hemianopia subsequent to infarction in the distribution of the posterior cerebral artery. Neuroophthalmology 11:33-39

5. Kerkhoff G, Munssinger U, Haaf E, Eberle-Strauss G, Stogerer E (1992) Rehabilitation of homonymous scotoma in patients with postgeniculate damage of the visual system: saccadic compensation training. Restor Neurol Neurosci 4:245-254

6. Kerkhoff G (1999) Restorative and compensatory therapy approaches in cerebral blindness-a review. Restor Neurol Neurosci 15:255-271

7. Weiskrantz L (2004) Roots of blindsight. Prog Brain Res 144:229-241

8. Pambakian ALM, Currie JC, Kennard C (2005) Rehabilitation strategies for patients with homonymous visual field defects. J Neuroophthalmol 25:136-142
9. Zihl J (1980) 'Blindsight': improvement of visually guided eye movements by systematic practise in patients with cerebral blindness. Neuropsychologia 18:71-77

10. Zihl J, von Cramon D (1980) Registration of light stimuli in the cortically blind hemifield and its effect on localisation. Behav Brain Res 1:287-298

11. Kasten E, Wuest S, Behrens-Baumann W, Sabal BA (1998) Computer-based training for the treatment of partial blindness. Nat Med 4:1083-1087

12. Kasten E, Poggel DA, Sabel BA (2000) Computer-based training of stimulus detection improves colour and simple pattern recognition in the defective field of hemianopic subjects. J Cogn Neurosci 12:1001-1012

13. Savir H (1977) Homonymous hemianopia and rehabilitation in fifteen cases of CCI. Scand J Rehabil Med 9:151-153

14. Sabel BA, Kenkel S, Kasten E (2005) Visual restoration therapy. Br J Ophthalmol 89:792-794

15. Schreiber A, Vonthein R, Reinhard J, Trauzettel-Klosinski S, Connert C, Sciefer U (2006) Effect of visual restitution training on absolute homonymous scotomas. Neurology 67:143-145

16. Henriksson L, Raninen A, Nasanen R, Hyvarinen L, Vanni S (2007) Training-induced cortical representation of a hemianopic hemifield. J Neurol Neurosurg Psychiatry 78:74-81

17. Balliet R, Blood KMT, Bach-Y-Rita P (1985) Visual field rehabilitation in the cortically blind? J Neurol Neurosurg Psychiatry 48:1113-1124

18. Bach-Y-Rita P (1983) Controlling variables eliminates hemianopsia rehabilitation results. Behav Brain Res 6:448

19. Pommerenke K, Markowitsch HJ (1989) Rehabilitation training of homonymous visual field defects in patients with postgeniculate damage of the visual system. Restor Neurol Neurosci 1:4763

20. Reinhard J, Schreiber A, Schiefer U, Kasten E, Sabel BA, Kenkel S, Vonthein R, Trauzettel-Klosinski S (2005) Does visual restitution training change absolute homonymous visual field defects? A fundus controlled study. Br J Ophthalmol 89:30-35

21. Horton JC (2005) Disappointing results from Nova Vision's visual restoration therapy. Br J Ophthalmol 89:1-2

22. Plant GT (2005) A work out for hemianopia. Br J Ophthalmol $89: 1-2$

23. Horton JC (2005) Vision restoration therapy: confounded by eye movements. Br J Ophthalmol 89:792-794

24. Zihl J (1995) Visual scanning behaviour in patients with homonymous hemianopia. Neuropsychologia 33:287-303

25. Meienberg O, Zangemeister WH, Rosenberg M, Hoyt WF, Stark L (1981) Saccadic eye movement strategies in patients with homonymous hemianopia. Ann Neurol 9:537-544

26. Pambakian A, Wooding DS, Patel N, Morland AB, Kennard C, Mannan SK (2000) Scanning the visual world: a study of patients with homonymous hemianopia. J Neurol Neurosurg Psychiatry 69:751-759

27. Zangemeister WH, Oechsner U, Freksa C (1995) Short term adaptation of eye movements in patients with visual hemi-field defects indicates high level control of human scanpaths. Optom Vis Sci 72:467-477

28. Nelles G, Esser J, Eckstein A, Tiede A, Gerhard H, Diener C (2001) Compensatory visual field training for patients with hemianopia after stroke. Neurosci Lett 306:189-192

29. Bolognini N, Rasi F, Coccia M, Ladvas E (2005) Visual search improvement in hemianopic patients after audio-visual stimulation. Brain 128:2830-2842

30. Pambakian A, Mannan SK, Hodgson TL, Kennard C (2004) Saccadic visual search training: a treatment for patients with homonymous hemianopia. J Neurol Neurosurg Psychiatry 75:1443-1448 
31. Kerkhoff G, Munssinger U, Meier K (1994) Neurovisual rehabilitation in cerebral blindness. Arch Neurol 51:474-481

32. Ishiai S, Furukawa T, Tsukagoshi H (1987) Eye-fixation patterns in homonymous hemianopia and unilateral spatial neglect. Neuropsychologia 25:675-679

33. Gassel MM, Williams D (1963) Visual function in patients with homonymous hemianopia. Part II. Oculomotor mechanisms. Brain 86:1-36

34. Behrmann M, Watt S, Black SE, Barton JJ (1997) Impaired visual search in patients with unilateral neglect: an oculographic analysis. Neuropsychologia 35:1445-1458

35. Passamonti C, Bertini C, Làdavas E (2009) Audio-visual stimulation improves oculomotor patterns in patients with hemianopia. Neuropsychololgia 47:546-555

36. Nelles G, Pscherer A, de Greiff A, Forsting M, Gerhard H, Esser J, Diener HC (2009) Eye-movement training-induced plasticity in patients with post-stroke hemianopia. J Neurol 256:726-733

37. Roth T, Sokolov AN, Messias A, Roth P, Weller M, TrauzettelKlosinski S (2009) Comparing explorative saccade and flicker training in hemianopia: a randomized controlled study. Neurology 72:324-331

38. Schuett S, Kentridge RW, Zihl J, Heywood CA (2009) Adaptation of eye-movements to simulated hemianopia in reading and visual exploration: transfer or specificity? Neuropsychologia 47:1712-1720

39. Barbur JL, Thompson WD, Forsyth PM (1987) A new system for the simultaneous measurements of pupil size and two-dimensional eye-movements. Clin Vis Sci 2:131-142

40. Wilson BJ, Cockburn, Halligan PW (1987) Behavioural inattention test. Bury St Edmunds, Thames Valley

41. Jacobs AM (1986) Le controle oculomteur dans l'exporation visuelle: mecanismes sensorimoteurs et processus cogitifs. Universite Rene Descartes, Paris

42. Poggio T, Fahle M, Edelman S (1992) Fast perceptual learning in visual hyperacuity. Science 256:1018-1021

43. Shoupes AA, Vogels R, Orban GA (1995) Human perceptual learning in identifying the oblique orientation:retinotopy, orientation specificity and monocularity. J Physiol 483:797-810

44. Karni A, Sagi D (1991) Where practice makes perfect in texture discrimination: evidence for primary visual cortex plasticity. Proc Natl Acad Sci 88:4966-4970

45. Ahissar M, Hochstein S (1993) Attentional control of early perceptual learning. Proc Natl Acad Sci USA 90:5718-5722
46. Ahissar M, Laiwand R, Kozminsky G, Hochstein S (1998) Learning pop-out detection: building representations for conflicting target-distractor relationships. Vision Res 38:3095-3107

47. Ahissar M, Hochstein S (1997) Task difficulty and the specificity of perceptual learning. Nature 387:401-406

48. Pambakian ALM, Kennard C (1997) Can visual function be restored in patients with homonymous hemianopia. $\mathrm{Br} \mathrm{J}$ Ophthalmol 81:324-328

49. Brant T, Thie A, Caplan LR, Hacke W (1995) Infarcts in the brain areas supplied by the posterior cerebral artery. Clinical aspects, pathogenesis and prognosis. Nervenarzt 66:267-274

50. Gray CS, French JM, Bates D, Cartlidge NE, Venables GS, James OF (1989) Recovery of visual fields in acute stroke:homonymous hemianopia associated with adverse prognosis. Age Ageing $18: 419-421$

51. Kalra L, Smith DH, Crome P (1993) Stroke in patients aged over 75 years: outcome and predictors. Postgrad Med J 69:33-36

52. Patel AT, Duncan PW, Lai SM, Studenski S (2000) The relation between impairments and functional outcomes poststroke. Arch Phys Med Rehabil 81:357-363

53. Han L, Law-Gibson D, Reding M (2002) Key neurological impairments influence function-related group outcomes after stroke. Stroke 33:1920-1924

54. Kalra L, Duncan PW, Lai SM, Studenski S (1997) The influence of visual neglect on stroke rehabilitation. Stroke 28:1386-1391

55. Cassidy TP, Bruce DW, Lewis S, Gray CS (1999) The association of visual field deficits and visuo-spatial neglect in acute righthemisphere stroke patients. Age Ageing 28:257-260

56. Cassidy TP, Lewis S, Gray CS (1998) Recovery from visuospatial neglect in stroke patients. J Neurol Neurosurg Psychiatry 64:555-557

57. Jehkonen MJ, Ahonen, Dastidar P (2000) Visual neglect as a predictor of functional outcome one year after stroke. Acta Neurol Scand 104:136-141

58. Raninen A, Vanni S, Hyvarinen S, Nasanen R (2007) Temporal sensitivity in a heminaopic visual field can be improved by long term training using flicker simulation. J Neurol Neurosurg Psychiatry 78:66-73

59. Jobke S, Kasten E, Sabel BA (2009) Visual restoration through extrastriate simulation in patients with visual field defects: a double-blind and randomized experimental study. Neurorehabil Neural Repair 23:246-255 